\title{
Dual-wavelength generation with terahertz spacing using GaAs-AIGaAs microring resonator waveguides
}

\begin{abstract}
In this research, we present the demonstration of GaAs-AlGaAs waveguide resonators. Two microring resonators (MRRs) have the same radius of $6.36 \mu \mathrm{m}$ are coupled and used to generate dual-wavelength with terahertz $(\mathrm{THz})$ spacing. We have shown that such resonators can be used to generate ultra-wide free spectral range (FSR) pulses with $\mathrm{THz}$ spacing, providing $\mathrm{THz}$ photonics communication signals. A Gaussian laser beam with power of $1 \mathrm{~W}$ is used as input. The MRRs are modeled by using GaAs-AlGaAs with GaAs core having refractive index of 3.368 surrounded by AlGaAs $(n=3.135)$. The drop port outputs of the MRRs system contains a dual-wavelength generated within $33 \mathrm{~nm}$ wavelength range, having a linewidth of $1.48(185.320 \mathrm{GHz})$ and FSR of $3.95 \mathrm{~nm}(500 \mathrm{GHz})$ which varies slightly along the wavelength. Results were generated using the time-domain travelling wave (TDTW) method and capable of modeling both active and passive photonic circuits.
\end{abstract}

Keyword: Dual-wavelength; GaAs-AlGaAs waveguide; THz spacing 